\title{
Removal of transcranial a.c. current stimulation artifact from simultaneous EEG recordings by superposition of moving averages
}

Link to publication record in Manchester Research Explorer

Citation for published version (APA):

Casson, A. J., \& Kohli, S. (2015). Removal of transcranial a.c. current stimulation artifact from simultaneous EEG recordings by superposition of moving averages. In host publication IEEE.

\section{Published in:}

host publication

\section{Citing this paper}

Please note that where the full-text provided on Manchester Research Explorer is the Author Accepted Manuscript or Proof version this may differ from the final Published version. If citing, it is advised that you check and use the publisher's definitive version.

\section{General rights}

Copyright and moral rights for the publications made accessible in the Research Explorer are retained by the authors and/or other copyright owners and it is a condition of accessing publications that users recognise and abide by the legal requirements associated with these rights.

\section{Takedown policy}

If you believe that this document breaches copyright please refer to the University of Manchester's Takedown Procedures [http://man.ac.uk/04Y6Bo] or contact uml.scholarlycommunications@manchester.ac.uk providing relevant details, so we can investigate your claim.

\section{OPEN ACCESS}




\title{
Removal of transcranial a.c. current stimulation artifact from simultaneous EEG recordings by superposition of moving averages
}

\author{
Siddharth Kohli and Alexander J. Casson, Member, IEEE
}

\begin{abstract}
Transcranial Current Stimulation (tCS) is a recent development for non-invasive brain stimulation. At present tCS introduces large artifacts into simultaneous EEG (electroencephalogram) recordings, which must be removed to enable brain changes from during the stimulation to be monitored. This paper presents a new approach for removing artifacts of transcranial Alternating Current Stimulation (tACS) from simultaneous EEG. Termed the Superposition of Moving Averages (SMA) our approach is independent of the number of EEG channels used and has a low computational complexity for use in real-time online artifact removal and data responsive stimulation. Compared to a Principal Component Analysis (PCA) approach our results show that SMA achieves superior artifact removal performance, especially with regards to the use of low channel count EEG devices.
\end{abstract}

\section{INTRODUCTION}

Transcranial Current Stimulation (tCS) is a recent development for non-invasive brain stimulation. Operating by injecting small amounts of current into the scalp via rubber electrodes enclosed in saline soaked sponges, it is a cheap, portable, and easy-to-use neuro-modulation approach [1]. Within tCS several different forms are available:

- Transcranial Direct Current Stimulation (tDCS) where a constant current is applied.

- Transcranial Random Noise Stimulation (tRNS) where a randomly generated current is applied.

- Transcranial Alternating Current Stimulation (tACS) where a sinusoidally oscillating current is applied.

Typically, currents below $2 \mathrm{~mA}$ are used and applied for durations of up to 20 minutes, in applications ranging from Schizophrenia [2] and Parkinson's Disease [3] to enhancing working memory [4].

The electroencephalogram (EEG) is the natural imaging complement to tCS stimulation as it is based upon recording voltages while tCS injects currents, and it has a high temporal resolution for observing the evolution of the brain state in response to transient stimulation. However, to date most tCS studies have not made use of simultaneous EEG recordings. Instead they have been constrained to a model of analysis which compares the post stimulus data with data recorded before stimulation, see Fig. 1, making no use of EEG data from during the stimulation. While tCS is in principle compatible with simultaneous EEG, due to the tCS electrode impedance the current injection causes a voltage potential on the head which manifests as an artifact in the EEG trace,

The authors are with the School of Electrical and Electronic Engineering, The University of Manchester, UK. Email: siddharth.kohli@postgrad.manchester.ac.uk, alex.casson@manchester.ac.uk.

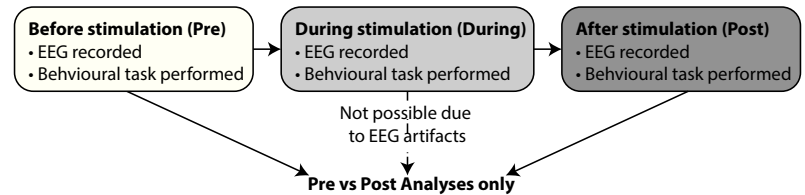

Fig. 1. EEG data can be recorded before (pre), during, and after (post) tCS stimulation. However, tCS artifacts in the during EEG data (Fig. 2) limit current studies to pre vs post analyses. New artifact removal procedures are required to enable pre vs during, and during vs post analyses.

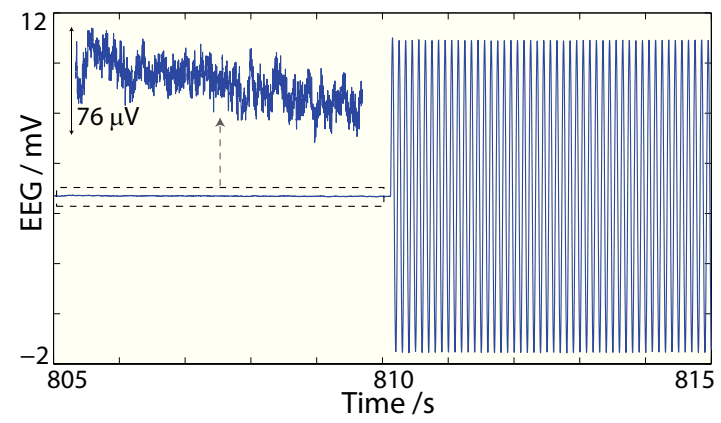

Fig. 2. Simultaneous EEG and tACS traces. Until $810 \mathrm{~s}$ good quality EEG is collected. After $810 \mathrm{~s}$ tACS stimulation starts and a large interference signal is seen. This artifact must be removed in order to allow the EEG from before, during, and after stimulation to be studied.

see Fig. 2. Further, tCS electrodes are physically largetypically up to $25 \mathrm{~cm}^{2}$-in order to keep the current density low (an essential safety criteria [5]). It limits the number of EEG electrodes that can be used, placing an emphasis on low channel count approaches. This is in addition to the need for keeping channel counts low when working with vulnerable subjects in order to keep the electrode set up time as short as possible.

To achieve an understanding of the underlying physiological activity during stimulation it is essential to devise new approaches for removing tCS artifacts from the EEG to enable analysis of the during stimulation EEG data. It has been investigated previously for tDCS simulation by using Independent Component Analysis (ICA) [6], [7]. This paper presents a new approach for removing tACS interference from simultaneous EEG recordings. Unlike the tDCS case where the artifacts take the form of systematic low frequency noise [6], tACS manifests as a sinusoidal signal (Fig. 2) at the stimulation frequency and its harmonics. When gamma $(>40 \mathrm{~Hz})$ stimulation is being applied, log scales can be used to separate tACS artifacts from true EEG as the stimulation is out of the band of most EEG studied $(0.1-30 \mathrm{~Hz})$ [8]. However, in general tACS stimulation frequencies overlap with cortical frequencies necessitating algorithmic separation. To date, one previous study has investigated the removal 
of tACS interference from simultaneous EEG [9]. based upon Principal Component Analysis (PCA) the authors were able to successfully show entrainment of neural oscillations using tACS, however no attempt was made to quantify the performance of the artifact removal algorithm.

We present a new approach based on moving-average filtering which we term Superposition of Moving Averages (SMA), Section II. Our results, Section III, demonstrate that this outperforms a PCA based approach. Further, SMA is formulated to be independent of the number of electrodes used and to have a low computational complexity for use in low channel count and real-time responsive stimulations based upon the current brain state.

\section{METHODS}

\section{A. Experimental procedure}

There were four subjects for this study, two male and two female, aged 23-31. The experiment was divided into six protocols with different stimulation settings, each three minutes long. As in Fig. 1 each protocol was divided into one minute long sections: Pre tACS stimulation; During tACS stimulation and Post tACS stimulation. In each one minute section the subject was asked to keep his/her eyes open for the first 28 seconds and closed for the next 32 seconds, with directions given verbally and in writing by an automated computer script. This approach allowed spontaneous alpha activity to be seen after the eyes were closed, giving a known brain state to be observed despite the presence of tACS artifact in the EEG.

For tACS stimulation two saline soaked sponge electrodes were placed on the head and connected to a battery-operated stimulator (DC-stimulator plus, Neuroconn, Germany). The target and reference electrodes were placed on the P3 and Fp4 to reflect montages used to target working memory performance in recent studies [4], [10]. Three different stimulation frequencies $(5,10$ and $40 \mathrm{~Hz}$ ) were used, and each of these was matched with a sham protocol where no stimulation was applied in the During period. This sham was single blinded and gave a total of six protocol runs (three different frequencies and three shams) for each subject. In all cases the stimulated current was $250 \mu \mathrm{A}$ peak-to-peak.

Simultaneous EEG was recorded using an 8 channel wireless EEG device (Enobio, Neuroelectrics, Spain). Electrode locations were determined using the ten-twenty system (Fp1, F3, F4, P3, P4, O1 and O2) with the reference and ground electrodes placed next to each other at $\mathrm{Cz}$ to provide a low channel count full head montage. All electrode impedances were maintained under $10 \mathrm{k} \Omega$ and EEG data was sampled at $500 \mathrm{~Hz}$. All procedures used in this study were reviewed and approved by the University of Manchester Research Ethics Committee.

\section{B. Artifact removal procedure}

1) SMA: The tACS interference signal manifests in all EEG channels. In each channel the amplitude of the interference is different, but the frequency and phase are global and periodic throughout the duration of the recording. This

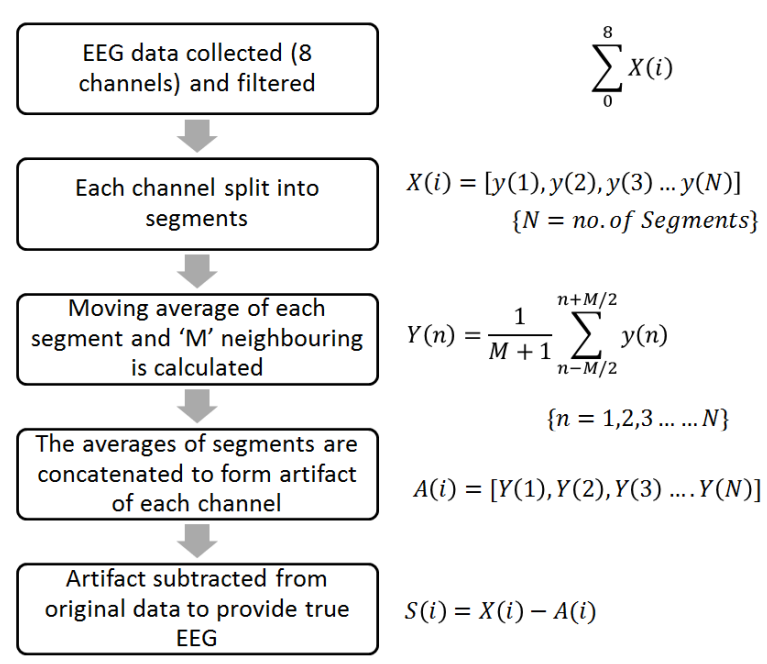

Fig. 3. Algorithm for SMA. Data from each channel is separately segmented before a moving average is calculated, which is then subtracted from the original data recorded for that channel.

is similar to the periodic artifacts seen in EEG data recorded during functional Magnetic Resonance Imaging (fMRI) and hence we base our new approach for tACS artifact removal on sliding moving averages, as has been applied in fMRI studies previously [11], [12]. The procedure, termed Superposition of Moving Averages (SMA), is illustrated in Fig. 3. The EEG data set, $X(i, t)$, where $i$ is the channel number and $t$ is time, is first split into non-overlapping segments such that the length of each segment matches the period of the tACS stimulation frequency. (If the period of stimulation cannot be split into segments with an integer number of samples the segment length is set to be as small as possible while also being periodic and an integer number of samples. For example, the segment length for one period of $40 \mathrm{~Hz}$ stimulation sampled at $500 \mathrm{~Hz}$ is 12.5 so 25 samples, two periods of the stimulation frequency, are used.)

Each segment, $y(i, n)$, where $n$ is the epoch index, and its $M$ neighboring segments are then central averaged to create a time and channel dependent local artifact template $A(i, n)$. This artifact template is subtracted from the data and the resulting signal after un-segmenting the signal, $S(i, t)$, represents the underlying EEG data during stimulation. As the artifact template is specific for each EEG channel this approach can map to an arbitrary number of channels without degrading performance. In this work $M$ is selected to be $5 \%$ of $N$ (the total number of segmented epochs) as a suitable trade-off between the number of averages taken and the time localization of the artifact template within the one minute stimulation period.

2) PCA: PCA constructs a set of weights, Principle Components (PC), based on the covariance of the EEG channels such that each component is uncorrelated with each other and that each component captures maximum variance of the dataset, excluding the variance captured by the previous component [13]. The first PC will have the maximum variance, that is, the most dominant component of the data, and each subsequent PC will have the next most 


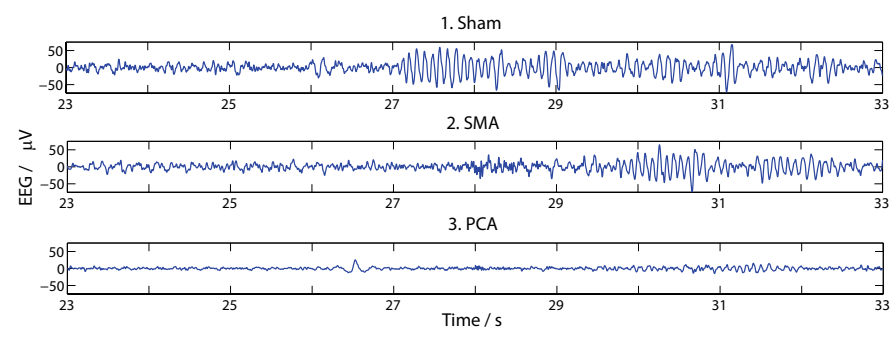

Fig. 4. Time domain plot of EEG data during sham (top) and after SMA (middle) and PCA (bottom) artifact removal. The subject is indicated to close his/her eyes after $28 \mathrm{~s}$ and an increase in alpha activity and alpha bursts are seen past this point. This is a representative illustration using data from one subject with $5 \mathrm{~Hz}$ tACS and EEG from $\mathrm{O} 2$.

dominant component of the data. As tACS artifact signals dominate over true EEG (Fig. 2) the artifact can be removed by decomposing the multi-channel EEG data set, $X(i, t)$, setting a number of the PCs to zero, and then reconstructing the EEG.

In this work no prior segmentation is applied and the entire one minute During section is decomposed by PCA as a single epoch. The PCs are calculated by representing the dataset of $I$ channels with each channel containing $T$ time samples as matrix $X_{I \times T}$. The covariance matrix of this is $R_{x x}$, with eigenvalues $\lambda_{1}, \lambda_{2}, \ldots \lambda_{I}\left(\lambda_{1} \geq \lambda_{2} \geq\right.$ $\ldots \lambda_{I}$ and corresponding eigenvectors $c_{1}, c_{2} \ldots c_{I}$. Also, $C=$ $\left[c_{1}, c_{2}, \ldots c_{I}\right]$ such that:

$$
C^{\prime} R_{x x} C=X_{I \times T}
$$

Each PC is given by $\lambda_{d} c_{d}$ and the reconstructed signal $S_{I^{*} \times T}$ is obtained as

$$
S_{I^{*} \times T}=\lambda_{i} c_{i} \times R_{d \times I} \times C^{\prime}
$$

where $d=[2,3, \ldots]$ (assuming only the first PC is removed) and $I^{*}$ is the number of PCs that are retained.

For low channel count approaches as considered here only the first PC is excluded as the remaining PCs may contain true EEG data. In the previous PCA study [9], there were 59 EEG channels and the first 3 PCs were excluded in the reconstruction.

\section{RESULTS AND DISCUSSION}

\section{A. Time domain analysis}

Fig. 4 illustrates EEG traces from $\mathrm{O} 2$ (where alpha is most dominant) when the subject closes his/her eyes after $\sim 28 \mathrm{~s}$. In the sham an increase in alpha activity and in alpha bursts is seen after this point, and this is observed in all of the protocols. After both SMA and PCA artifact removal the bursts of alpha are also seen in the reconstructed datasets for EEG during tACS. (Recall that the sham and artifact removed data are from different experimental runs. It is not expected that the SMA/PCA traces match the sham trace exactly, instead they should show the same pattern of alpha and alpha bursts once the eyes are closed. Both the SMA and PCA are applied to the same experimental run and so they would match exactly if both removed the same artifact.) The bursts of alpha are less apparent after PCA artifact removal compared to SMA. This is most likely due to the low channel
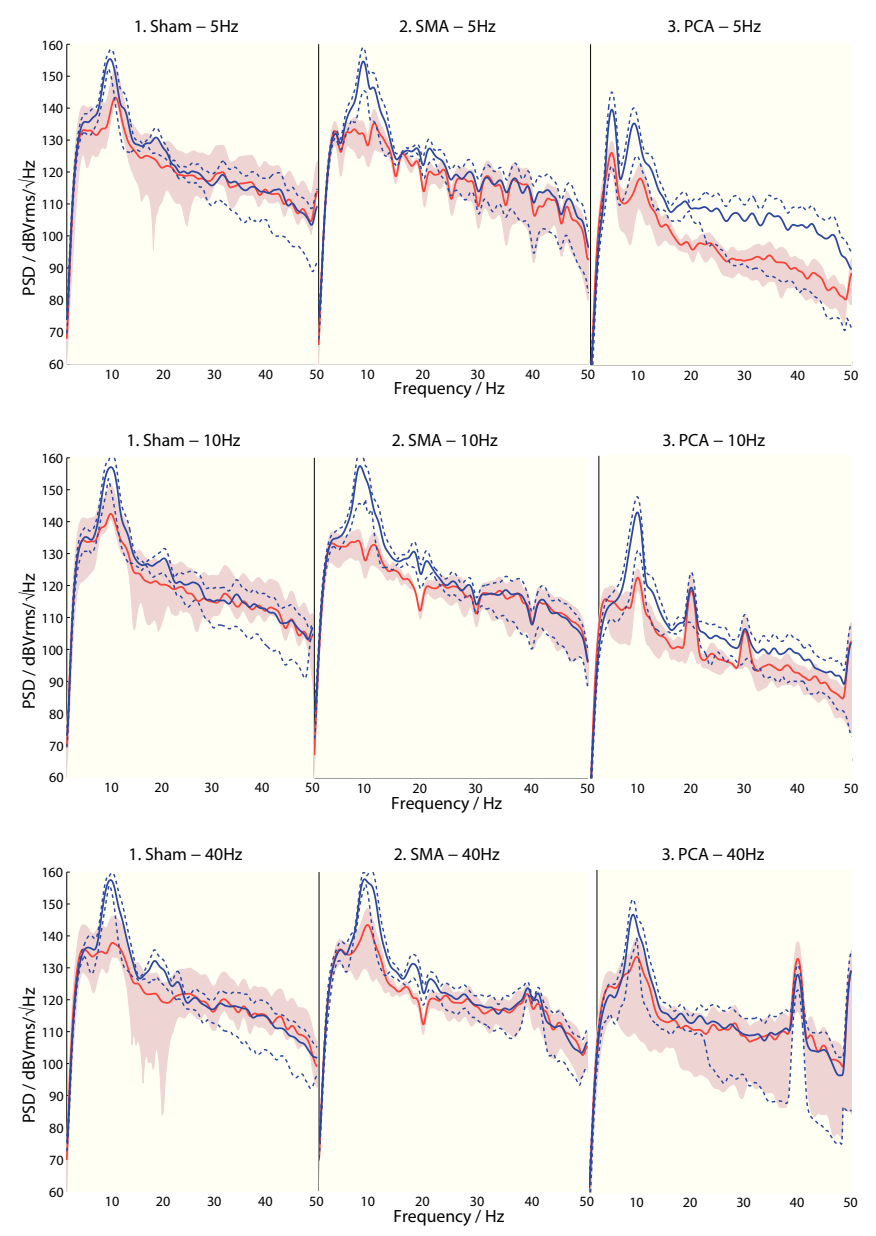

Fig. 5. Frequency domain information in channel $\mathrm{O} 2$ averaged across subjects with interquartile ranges. Sham (left), SMA artifact removed (middle) and PCA artifact removed (right) with eyes open (red lines) and eyes closed (blue lines).

EEG signals considered in this study such that the first PC contains some components of the underlying EEG. This can be countered by using EEG montages with greater number of channels, which has its own drawbacks (Section IV). In contrast the SMA approach is independent of the number of channels and clear alpha bursts are seen shortly after the eyes are closed.

\section{B. Frequency domain analysis}

Fig. 5 shows the EEG frequency domain information from the During stimulation periods, averaged across the subjects. The increase in alpha power when the eyes are closed (blue line) is clearly seen for all protocols, demonstrating the potential for alpha tracking and responsive matched frequency/non-matched frequency stimulation (see Section III-C). This said, two non-ideal effects are seen in the reconstructed signals.

Firstly, when the eyes are open (red lines) the alpha activity is much lower in the $5 \mathrm{~Hz}$ and $10 \mathrm{~Hz}$ tACS stimulation cases (using both the SMA and PCA artifact removal processes) compared to the sham. This is not observed in the $40 \mathrm{~Hz}$ stimulation case where the alpha power is approximately the same in the sham and artifact removed cases. It 
TABLE I

IAFS FOR ALL SUBJECTS DURING $40 \mathrm{HZ}$ TACS STIMULATION SHOW A CHANGE BETWEEN EYES OPEN AND CLOSED IN BOTH SHAM AND STIMULATION PROTOCOLS. Eyes OPEN (EO), Eyes Closed (EC).

\begin{tabular}{|c|c|c|c|c|c|c|}
\hline & \multicolumn{2}{|c|}{ Pre } & \multicolumn{2}{|c|}{ During } & \multicolumn{2}{|c|}{ Post } \\
\hline & EO & $\mathrm{EC}$ & EO & EC & EO & EC \\
\hline Sham & 09.79 & 10.00 & 09.36 & 09.53 & 10.31 & 09.76 \\
\hline $\begin{array}{l}\text { PCA } \\
\text { SMA }\end{array}$ & 10.59 & 10.04 & $\begin{array}{l}09.34 \\
09.39\end{array}$ & $\begin{array}{l}09.75 \\
09.76\end{array}$ & 08.37 & 09.91 \\
\hline Sham & 09.88 & 10.03 & 08.32 & 10.11 & 11.09 & 10.04 \\
\hline $\begin{array}{l}\text { PCA } \\
\text { SMA }\end{array}$ & 09.76 & 10.13 & $\begin{array}{l}09.32 \\
09.35\end{array}$ & $\begin{array}{l}10.46 \\
10.51\end{array}$ & 10.61 & 10.19 \\
\hline Sham & 10.23 & 09.26 & 09.95 & 09.30 & 09.28 & 09.21 \\
\hline $\begin{array}{l}\text { PCA } \\
\text { SMA }\end{array}$ & 09.68 & 09.14 & $\begin{array}{l}09.97 \\
09.94\end{array}$ & $\begin{array}{l}09.22 \\
09.20\end{array}$ & 10.05 & 09.18 \\
\hline Sham & 11.71 & 08.94 & 10.49 & 08.98 & 09.27 & 09.02 \\
\hline $\begin{array}{l}\text { PCA } \\
\text { SMA }\end{array}$ & 10.55 & 09.10 & $\begin{array}{l}10.60 \\
09.88\end{array}$ & $\begin{array}{l}09.13 \\
09.13\end{array}$ & 10.08 & 09.02 \\
\hline
\end{tabular}

suggests that the artifact removal process is removing some physiological information when the stimulation frequency is close to the frequency to be observed. Both the SMA and PCA removal processes have this limitation when analyzing background EEG. Nevertheless, tACS is known to enhance alpha activity, and the bursts of alpha observed in Fig. 4, together with the substantial increase in alpha activity when eyes are closed (Fig. 5) demonstrate that elevated EEG bands under tACS can be studied without issue.

Secondly, residual interference at the harmonics of the stimulation frequency can be seen after the PCA removal process, most apparent for the $10 \mathrm{~Hz}$ stimulation results. These are large artifacts, with the same magnitude in the eyes open and eyes closed cases indicating that they are unlikely to be of neurophysiological origin. In contrast, there is a dip at these frequencies after SMA (Fig. 5-middle) artifact removal. In both the sham and SMA results a small increase in activity around $20 \mathrm{~Hz}$ is observed when the eyes are closed, but this cannot be observed after PCA artifact removal due to the presence of the residual stimulation harmonics. This points towards SMA being the superior of the two artifact removal methods. In our experiments to date, the removal of the stimulation harmonics in addition to the fundamental frequency has been a substantial challenge. The SMA approach is capable of eliminating these harmonics, although as seen it also results in the loss of some physiological information at the stimulation frequency.

\section{Individual alpha tracking}

Finally, to demonstrate the potential for individual responsive stimulation we show the Individual Alpha Frequency (IAF) as another measure of accuracy of the reconstructed EEG data [14]. Table I shows how the IAF varies during the $40 \mathrm{~Hz}$ tACS protocols for all four subjects. It is important note that a distinct change in IAF during stimulation when eyes are closed is seen consistently for all subjects, and that this change matches the change in IAF during sham protocols. This allows us to further conclude that the reconstructed data accurately demonstrates physiological EEG data during tACS stimulation.

\section{CONCLUSIONS}

The only other study [9] to formally investigate tACS artifact removal used PCA with a 59 channel EEG montage. We have presented tACS artifact removal using only a single EEG channel. This allows for much lower channel EEG+tACS experiments to be implemented, providing the benefits of: allowing cheaper lower channel count EEG devices to be used; enabling EEG montages with space for the physically large tACS electrodes (especially important with emerging high-definition tACS (HD-tACS) where multiple tACS electrodes are used to improve stimulation focality); and quicker experimental set-ups which can be crucial for large scale studies with vulnerable subjects in the future.

\section{ACKNOWLEDGMENTS}

The authors would like to acknowledge and thank Dr Ira Leroi for enabling the experimental aspects of this work.

\section{REFERENCES}

[1] C. S. Herrmann, S. Rach, T. Neuling, and D. Struber, "Transcranial alternating current stimulation: A review of the underlying mechanisms and modulation of cognitive processes," Front. Hum. Neurosci., vol. 7, no. $279,2013$.

[2] S. M. Agarwal, V. Shivakumar, A. Bose, A. Subramaniam, H. Nawani, H. Chhabra, S. V. Kalmady, J. C. Narayanaswamy, and G. Venkatasubramanian, "Transcranial direct current stimulation in schizophrenia," Clin. Psychopharmacol. Neurosci., vol. 11, no. 3, pp. 118-125, 2013.

[3] J.-S. Brittain, P. Probert-Smith, T. Aziz, and P. Brown, "Tremor suppression by rhythmic transcranial current stimulation," Curr. Biol., vol. 23, no. 5, pp. 436-440, 2013.

[4] N. Jausovec and K. Jausovec, "Increasing working memory capacity with theta transcranial alternating current stimulation (tACS)," Biol. Psychol., vol. 96, pp. 42-47, 2014.

[5] P. C. Gandiga, F. C. Hummel, and L. G. Cohen, "Transcranial DC stimulation (tDCS): A tool for double-blind sham-controlled clinical studies in brain stimulation," Clin. Neurophysiol., vol. 117, no. 4, pp. 845-850, 2006.

[6] A. Roy, B. Baxter, and B. He, "High-definition transcranial direct current stimulation induces both acute and persistent changes in broadband cortical synchronization: A simultaneous tDCS-EEG study," IEEE Trans. Biomed. Eng., vol. 61, no. 7, pp. 1967-1978, 2014.

[7] B. S. Baxter, B. Edelman, X. Zhang, A. Roy, and B. He, "Simultaneous high-definition transcranial direct current stimulation of the motor cortex and motor imagery," in IEEE EMBC, Chicago, August 2014.

[8] U. Voss, R. Holzmann, A. Hobson, W. Paulus, J. Koppehele-Gossel, A. Klimke, and M. A. Nitsche, "Induction of self awareness in dreams through frontal low current stimulation of gamma activity," Nature Neurosci., vol. 17, no. 6, pp. 810-812, 2014.

[9] R. A. F. Helfrich, T. R. Schneider, S. Trautmann-Lengsfeld, A. Engel, and C. Herrmann, "Entrainment of brain oscillations by transcranial alternating current stimulation," Curr. Biol., vol. 24, no. 3, pp. 333339, 2014.

[10] O. Meiron and M. Lavidor, "Prefrontal oscillatory stimulation modulates access to cognitive control references in retrospective metacognitive commentary," Clin. Neurophysiol., vol. 125, no. 1, pp. 77-82, 2014.

[11] P. J. Allen, O. Josephs, and R. Turner, "A method for removing imaging artifact from continuous EEG recorded during functional MRI," NeuroImage, vol. 12, no. 2, pp. 230-239, 2000.

[12] R. K. Niazy, C. F. Beckmann, G. D. Iannetti, J. M. Brady, and S. M. Smith, "Removal of fMRI environment artifacts from EEG data using optimal basis sets," NeuroImage, vol. 28, no. 3, pp. 720-737, 2005.

[13] M. X. Cohen, Analyzing Neural Time Series Data. Cambridge, Massachusetts: The MIT Press, 2014.

[14] M. Arns, W. H. Drinkenburg, P. B. Fitzgerald, and J. L. Kenemans, "Neurophysiological predictors of non-response to rTMS in depression,” Brain Stimul., vol. 5, no. 4, pp. 569-576, 2012. 